\title{
A Neutrophil activation signature in Covid-19
}

\author{
Athanasios Didangelos (PhD) \\ ad482@leicester.ac.uk

\section{University of Leicester, Mayer IgA Nephropathy Laboratory, University of Leicester, Leicester, LE1 7RH, United Kingdom}

\begin{abstract}
Covid-19 is often related to hyperinflammation that drives lung or multi-organ damage and mortality. The immunopathological mechanisms that cause excessive inflammation following SARS-Cov-2 infection are under investigation while different approaches to limit hyperinflammation in affected patients are being proposed. Here, a computational network approach was used on recently available data to identify possible Covid-19 inflammatory mechanisms. First, network analysis of putative SARS-Cov-2 cellular receptors and their directly associated interacting proteins, led to the mining of a robust neutrophil-response signature and multiple relevant inflammatory response genes. Second, analysis of RNA-seq datasets of lung epithelial cells infected with SARS-Cov-2 found that infected cells specifically expressed neutrophil-attracting chemokines, further supporting the likely role of neutrophils in Covid-19 inflammation. The role of neutrophils in Covid-19 needs to be studied further. Different immunoregulatory molecules and pathways presented here (TNF Receptor, IL8, CXCR1, CXCR2, ADAM10, GPR84, MME-neprilysin, ANPEP, LAP3) are druggable and might be therapeutic targets in efforts to limit SARS-Cov-2 inflammation in severe clinical cases.
\end{abstract}

\section{Introduction}

New studies have highlighted that Covid-19 is often characterised by an extreme inflammatory response associated with lung and multi-organ injury and mortality and have suggested promising anti-inflammatory options (1). Other studies recommend caution with immunosuppression given that regulated inflammation is necessary for an effective anti-viral response (2). More ideas are needed to understand Covid-19 hyperinflammation and target relevant immunopathological mechanisms. The role and function of different myeloid cell types in Covid-19 lung injury, cytokine storm and sepsis are not well understood.

\section{Methods}

The data used for the analysis was from publicly available published sources as cited below. Lung epithelial cell RNA-seq data was downloaded from https://www.biorxiv.org/content/10.1101/2020.03.24.004655v1.supplementary-material

(Supplemental Table 2). Only genes significant by adjusted $p$-value were included. Network analysis was performed using StringDB. For the 7 coronavirus receptors network inflation was performed on StringDB by adding 100 first-shell (direct first-degree protein interactions). For the lung epithelial cell RNA-seq data only the 115 significant proteins were used to make the protein-protein interaction network. Network cut-off was set at 0.4. Gene Ontology analysis was performed using GeneOntology and validated in BiNGO in Cytoscape Drug-Gene interactions were analysed and downloaded from DGIdb. Network analysis, comparisons and final designs were performed on Cytoscape.

\section{Results and Discussion}

A recent study identified 7 putative SARS-Cov-2 receptors including the now well-known ACE2 (3) together with peptidases DPP4 and ANPEP as well as pathogen-binding proteins CD209, CLEC4G, CLEC4M and CEACAM1 (Fig.1A). These 7 proteins are expressed in different cell types and are implicated in the binding of coronaviruses on epithelial cells (4). To examine these 7 proteins further, I used freely-accessible network analysis (StringDB). The small signature (Fig.1A) was inflated to include up to 100 proteins that directly interact with the 7 input proteins (Fig.1B). Network inflation was performed to identify proteins that directly associate with the putative coronavirus receptors on human cells, thereby allowing mining of 
possible functional pathways. Surprisingly, the main ontology of this expanded network was "Neutrophil Degranulation" (GO:0043312) with 70 proteins in this category (Fig.1C). There were also 8 neutrophil-specific genes (Neutrophil-specific genes (5) including ANPEP, MME, MGAM, CD177, CEACAM1/3, FPR2 and CYSTM1 (Fig.1D). This finding suggested that coronavirus binding proteins (and their direct interactors) might be involved in neutrophil or other related classic inflammatory mechanisms. Two of the neutrophil-specific genes, ANPEP and CEACAM1 (Fig.1D) are coronavirus receptors (Fig.1A) indicating that neutrophils could be infected by SARS-Cov-2, as they are by Influenza A (6). At the time of writing this paper, interaction or infection of neutrophils with SARS-Cov-2 had not been reported.

The role of neutrophils in viral infections of the upper respiratory tract and their possible involvement in therapeutic strategies is not entirely clarified (7). They are involved in early anti-viral defence (8) but through degranulation and lysis, they can also be cytotoxic during ARDS and severe pneumonia, including from coronaviruses (9). Neutrophil hyperinflammation is also likely in other severe viral infections such as Hepatitis (7). In current Covid-19 literature, an increased peripheral neutrophil-to-lymphocyte ratio is observed in severe cases (10) but not much is known regarding neutrophils in Covid-19 affected lungs. Covid-19 lung injury in specific patients might involve dysregulated neutrophil activity.

To examine neutrophil recruitment from the perspective of Covid-19, I analysed a publicly available RNA-seq dataset of human lung epithelial cells infected with SARS-Cov-2 (11), to find gene expression changes following SARS-Cov-2 infection. Regulated genes are presented in (Fig.1E). As expected, the gene signature contains 39 inflammation and viral response genes, including classic inflammatory mediators and interferon pathway genes (Fig.1F). Notably, the SARS-Cov-2 infected lung epithelial cells overexpressed 6 chemokines that belong to the human ontology annotation "Neutrophil Chemotaxis" (GO:0030593), and include the classic neutrophil chemoattractants CXCL1, CXCL2, CXCL3, CXCL5, IL8 (CXCL8) and CCL20 (Fig.1G), suggesting that these cells can express neutrophil chemokines after SARS-Cov-2 infection. The receptors for these chemokines (CXCR2 and CXCR1; IL8 receptor) are neutrophil-specific genes like CXCL1, CXCL5, IL8, ANPEP and CEACAM1 (Neutrophil-specific genes). SARS-Cov-2 infected cells also overexpressed complement C3 and associated pathway activation genes (Fig.1H), while the receptor for the C3a anaphylatoxin (C3AR1) is a "Neutrophil Degranulation" gene (Fig.1B). C3 and complement activation have been recently involved in ARDS with systemic inflammation and lung neutrophilia (12).

Are neutrophils and related inflammatory mechanisms likely targets in Covid-19 complications? This is a difficult question given the complexity of acute innate immune responses and the importance of neutrophils in early anti-viral defence. Universal suppression of neutrophils or other myeloid cell types is not trivial and the clinical evidence on the use of steroids in Covid-19 inconclusive. Nevertheless, it might be possible to target specific inflammatory mechanisms before ARDS. The target-mining presented here includes multiple druggable proteins (Fig.1I-K) such as neutrophil-attracting chemokine signalling (Fig.1I), neutrophil-relevant inflammatory entities (Fig.1J) and SARS-Cov-2 receptors (Fig.1K).

IL8 (Fig.1I) a cardinal neutrophil chemoattractant and product of activated neutrophils, can be blocked by neutralising antibodies (HuMax-IL8; FDA/EMA-approved). The neutrophil chemokine receptors CXCR1 and CXCR2 are also targeted by different experimental drugs including the approved CXCR2 blocker AZD5069 (Fig.1H). Other interesting inflammatory proteins include the monocyte and neutrophil-enriched genes TNF-receptor-2 (TNFR2 or TNFRSF1B), GPR84 and ADAM10 (Fig.1J). TNFR2 is clinically blocked by the receptorantagonist Enbrel. While TNF has a well-established role in neutrophil activation and prolongs neutrophil survival (13), the role of TNF in Covid-19 infection has not received attention. GPR84 and ADAM10 are targeted with experimental drugs including approved PBI-4050 (Setogepram) and the metzincin inhibitor llomastat respectively (Fig.1J). FPR2 (formyl 
peptide receptor), MME (neprilysin) and MGAM (maltase-glucoamylase) are directly involved in inflammatory neutrophil recruitment and activity and are neutrophil-specific genes (Fig.1D). ANPEP (Aminopeptidase-N), a likely coronavirus receptor (Fig.1A) and a neutrophil-specific gene (Fig.1D), is potentially blocked by approved drugs Ezetimibe and Icatibant, while there is experimental evidence that it interacts with Tosedostat and Ubenimex. It is however unlikely that binding of these drugs to the aminopeptidase will interfere with viral binding. Ubenimex is also an inhibitor of LAP3 (leucine aminopeptidase-3), the only gene shared between the coronavirus receptors inflated network (Fig.1A) and SARS-Cov-2 infected lung epithelial cells (Fig.1F). LAP3 was induced in lung epithelial cells following SARS-Cov-2 infection and it is a monocyte and neutrophil-enriched gene. Like other aminopeptidases (like ANPEP), LAP3 might be another coronavirus receptor.

\section{Conclusions}

Neutrophil recruitment and related inflammatory activity might be important components of severe Covid-19 immunopathology. In contrast to universal immunosuppression, specific inflammatory proteins and pathways (chemokine receptors, TNFR, neprilysin, FPR2, complement C3) could be considered in patients suffering from Covid-19 hyperinflammation. Moreover, the role of neutrophils in recognition of SARS-Cov-2 and the possibility that they are infected with the virus needs to be studied further. Finally, it is perhaps worth noting that some currently investigated Covid-19 therapeutic options include hydroxychloroquine, azithromycin and colchicine. All three have well-studied anti-neutrophil effects (14-16).

\section{References}

1. Mehta P, McAuley DF, Brown M, Sanchez E, Tattersall RS, Manson JJ, HIh Across Speciality Collaboration UK. 2020. COVID-19: consider cytokine storm syndromes and immunosuppression. Lancet 395:1033-1034.

2. Ritchie AI, Singanayagam A. 2020. Immunosuppression for hyperinflammation in COVID-19: a double-edged sword? Lancet 395:1111.

3. Ou X, Liu Y, Lei X, Li P, Mi D, Ren L, Guo L, Guo R, Chen T, Hu J, Xiang Z, Mu Z, Chen X, Chen J, Hu K, Jin Q, Wang J, Qian Z. 2020. Characterization of spike glycoprotein of SARS-CoV-2 on virus entry and its immune cross-reactivity with SARSCoV. Nat Commun 11:1620.

4. Qi F, Qian S, Zhang S, Zhang Z. 2020. Single cell RNA sequencing of 13 human tissues identify cell types and receptors of human coronaviruses. Biochem Biophys Res Commun doi:10.1016/j.bbrc.2020.03.044.

5. Uhlen M, Karlsson MJ, Zhong W, Tebani A, Pou C, Mikes J, Lakshmikanth T, Forsstrom B, Edfors F, Odeberg J, Mardinoglu A, Zhang C, von Feilitzen K, Mulder J, Sjostedt E, Hober A, Oksvold P, Zwahlen M, Ponten F, Lindskog C, Sivertsson A, Fagerberg L, Brodin P. 2019. A genome-wide transcriptomic analysis of protein-coding genes in human blood cells. Science 366.

6. Hufford MM, Richardson G, Zhou H, Manicassamy B, Garcia-Sastre A, Enelow RI, Braciale TJ. 2012. Influenza-infected neutrophils within the infected lungs act as antigen presenting cells for anti-viral CD8(+) T cells. PLoS One 7:e46581.

7. Drescher B, Bai F. 2013. Neutrophil in viral infections, friend or foe? Virus Res 171:17.

8. Camp JV, Jonsson CB. 2017. A Role for Neutrophils in Viral Respiratory Disease. Front Immunol 8:550.

9. Haick AK, Rzepka JP, Brandon E, Balemba OB, Miura TA. 2014. Neutrophils are needed for an effective immune response against pulmonary rat coronavirus infection, but also contribute to pathology. J Gen Virol 95:578-590.

10. Zheng M, Gao Y, Wang G, Song G, Liu S, Sun D, Xu Y, Tian Z. 2020. Functional exhaustion of antiviral lymphocytes in COVID-19 patients. Cell Mol Immunol doi:10.1038/s41423-020-0402-2. 
11. Blanco-Melo D, Nilsson-Payant BE, Liu W-C, Møller R, Panis M, Sachs D, Albrecht RA, tenOever BR. 2020. SARS-CoV-2 launches a unique transcriptional signature from in vitro, ex vivo, and in vivo systems. bioRxiv doi:10.1101/2020.03.24.004655:2020.03.24.004655.

12. Gralinski LE, Sheahan TP, Morrison TE, Menachery VD, Jensen K, Leist SR, Whitmore A, Heise MT, Baric RS. 2018. Complement Activation Contributes to Severe Acute Respiratory Syndrome Coronavirus Pathogenesis. mBio 9.

13. Colotta F, Re F, Polentarutti N, Sozzani S, Mantovani A. 1992. Modulation of granulocyte survival and programmed cell death by cytokines and bacterial products. Blood 80:2012-20.

14. Cronstein BN, Molad Y, Reibman J, Balakhane E, Levin RI, Weissmann G. 1995. Colchicine alters the quantitative and qualitative display of selectins on endothelial cells and neutrophils. J Clin Invest 96:994-1002.

15. Culic O, Erakovic V, Cepelak I, Barisic K, Brajsa K, Ferencic Z, Galovic R, Glojnaric I, Manojlovic Z, Munic V, Novak-Mircetic R, Pavicic-Beljak V, Sucic M, Veljaca M, ZanicGrubisic T, Parnham MJ. 2002. Azithromycin modulates neutrophil function and circulating inflammatory mediators in healthy human subjects. Eur $\mathrm{J}$ Pharmacol 450:277-289.

16. Hurst NP, French JK, Gorjatschko L, Betts WH. 1988. Chloroquine and hydroxychloroquine inhibit multiple sites in metabolic pathways leading to neutrophil superoxide release. J Rheumatol 15:23-7.

\section{Figure Legend}

Fig.1: A: ACE2 and 6 related putative SARS-Cov-2 receptors on epithelial cells. ACE2, DPP4 and ANPEP are peptidases. B-D: The 7 putative SARS-Cov-2 receptors were inflated in StringDB $(v 10)$ by adding up to 100 directly interacting proteins with definite association (B). The main gene ontology of the inflated network is "Neutrophil Degranulation" (C) and included 8 Neutrophil-specific genes (D). E-H: 113 genes that were differentially regulated (RNA-seq) in lung epithelial cells following stimulation with SARS-Cov-2 (E). Following virus infection, most upregulated genes were related to inflammatory and virus responses $(\mathbf{F})$. 6 classic neutrophil chemokines were upregulated (G). SARS-Cov-2 infected epithelial cells also upregulated $\mathrm{C} 3$ and related complement pathway genes $(\mathbf{H})$. I-K: Putative druggable targets with neutrophil and inflammatory function derived from the analysis. Approved and experimental drugs with solid pharmacological evidence are presented as interaction networks. Protein-Drug associations were examined in DGiDB and were manually curated to exclude false-positive or non-experimentally validated interactions. All protein-protein interaction networks were performed in Cytoscape using interaction data derived from StringDB(v10). 
